\title{
Pronator Quadratus to Extensor Carpi Radialis Brevis Nerve Transfer in C5-C7 or C5-C8 Brachial Plexus Injuries for Independent Wrist Extension
}

\author{
Anil Bhatia ${ }^{1}$ Mahmoud Salama \\ ${ }^{1}$ Department of Brachial Plexus Surgery, Deenanath Mangeshkar \\ Hospital, Pune, India \\ 2Department of Orthopedics, Aswan University, Aswan, Egypt \\ Indian J Plast Surg 2020;53:36-41
}

\begin{abstract}
Address for correspondence Mahmoud Salama, MD, Faculty of Medicine, Aswan University, Aswan, Egypt (e-mail: mahmoudsalamaegy1@yahoo.com).
\end{abstract}

\begin{abstract}
Background Patients with lesions affecting C7 and C8 roots (in addition to C56) demonstrate loss of independent wrist dorsiflexion in addition to loss of shoulder abduction and elbow flexion. Traditionally, this deficit has been addressed using tendon transfers after useful function at the shoulder and elbow has been restored by primary nerve surgery. Confidence with nerve transfer techniques has prompted attempts to replace this method by incorporating procedures for wrist dorsiflexion in the primary operation itself.

Aim The objective of this study was to report the results of pronator quadratus motor branch transfers to the extensor carpi radialis brevis motor branch to reconstruct wrist extension in C5-C8 root lesions of the brachial plexus.

Patients and Methods Twenty-three patients, average age 30 years, with C5-8 root injuries underwent operations an average of 4.7 months after their accident. Extrinsic extension of the fingers and thumb was weak or absent in two cases while the remaining 18 patients could open their hand actively. The patients lacked independent wrist extension when they were examined with the fingers flexed as the compensatory action of the extrinsic finger extensors was removed. The average follow-up was 21 months postoperative with the minimal follow-up period was at least 12 months.

Keywords

- pronator quadratus

- nerve transfer

- brachial plexus injuries

- wrist extension

reconstruction

Results Successful reinnervations of the extensor carpi radialis brevis (ECRB) were demonstrated in all patients. In 17 patients, wrist extension scored M4, and in 3 patients it scored M3.

Conclusions The pronator quadratus (PQ) to ECRB nerve transfer in C5-C7 or C5-C8 brachial plexus injuries for independent wrist extension reconstruction gives consistently good results with minimal donor morbidity.
\end{abstract}

\section{Introduction}

The classification of incomplete brachial plexus palsies in terms of the affected roots has been a subject of debate till now. ${ }^{1}$ Neurological deficits with C5-6 root injuries are clearly limited to loss of shoulder abduction, external rotation, and of elbow flexion (supraspinatus, infraspinatus, biceps, brachialis, brachioradialis, deltoid, and teres minor). However, when weakness of other muscles, too, is noted the involvement of the $\mathrm{C} 7 \pm \mathrm{C} 8$ roots is suspected. ${ }^{2}$ While the exact contribution of each root is uncertain, the independent wrist extension is often deficient in those cases with extended upper brachial plexus injuries. Traditionally, the deficit at the shoulder and elbow is addressed during published online April 22, 2020
DOI https://doi.org/ 10.1055/s-0040-1708114 ISSN 0970-0358.
(C) 2020 Association of Plastic Surgeons of India
License terms

(c) (1) $\ominus \circledast$ 
primary nerve reconstruction while the wrist extension restoration is managed by a secondary tendon transfer in a second stage. ${ }^{3}$ Thus, such patients require at least two separate operative procedures and rehabilitation continues for at least 18 months till the final function achieved. The possibility of primary nerve reconstruction of the wrist extensors has been rarely studied in literature. Use of the tiny branches of the median nerve for the individual slips of the flexor digitorum superficialis does not seem appealing as that would weaken the grip strength and eliminate that option for a secondary tendon transfer in cases of failure of the nerve transfer. ${ }^{4}$ Bertelli's description of the use of the anterior interosseous nerve (AIN) to innervate the extensor carpi radialis brevis indicates a method that can be safely incorporated in the primary procedure. ${ }^{5}$

The current study hypothesis was that the pronator quadratus (PQ) to extensor carpi radialis brevis (ECRB) nerve transfer in $\mathrm{C} 5-\mathrm{C} 7$ or $\mathrm{C} 5-\mathrm{C} 8$ brachial plexus injuries is the best method for independent wrist extension reconstruction.

\section{Methods and Materials}

This cross-sectional descriptive study included 23 patients with extensive partial brachial plexus palsies were treated by primary nerve reconstruction surgery by the first author. Patients were recruited from the brachial plexus unit, in the period between March 2013 and December 2015.

Of these 23 cases, follow-up data was available for 20 patients. Each of these patients had suffered the injury in a motorcycle accident. The associated skeletal injuries had been dealt with before the brachial plexus intervention. The deficit in the upper limb was evaluated clinically. Involvement of $\mathrm{C} 7 / \mathrm{C} 8$ roots could be inferred based on absent function in the latissimus dorsi, and weak or absent pectoralis major, triceps, flexor carpi radialis, and flexor carpi ulnaris muscles. Extrinsic extension of the fingers and thumb was weak or absent in two cases while the remaining 18 patients could open their hand actively. Wrist extensor paralysis was determined by the wasting of the radial forearm muscles and by the inability to extend the wrist without simultaneous extension of the fingers and thumb. In addition, we looked for contraction at the tendon insertions at the bases of the second and third metacarpals. Extrinsic flexion and intrinsics were preserved in each case although the function of the flexor pollicis longus and flexor digitorum profundus to the index finger power were M3 at the time of the operation in six cases.

Grade 4 strength was noted when pronation was tested with the elbow and forearm supported. In one patient, the forearm was stiff in the neutral position due to the original injury. Integrity of the function was checked preoperatively by examining the thumb and index flexion, which scored M5 in 4 patients, M4 in 10 patients, and M3 in 6 patients. Finger extension reported to be $\geq \mathrm{M} 3$ in 18 cases, M2 in 1 case, and M0 in 1 case.

All patients had M0 of wrist extensor. Wrist extension was examined with flexed fingers to negate the compensatory action of the extrinsic extensors.
Magnetic resonance imaging (MRI) of the cervical spine was performed in each case. Surgery was proposed based on signs of root avulsions in combination with clinical features of extensive supraclavicular injuries.

A strong Tinel sign in the C5 territory was noted in some patients on application of pressure at the posterior border of the sternocleidomastoid muscle. The surgical strategy was based upon the available sources of growing axons and was decided upon after formal exploration of the roots at the intervertebral foramina.

\section{Operative Details}

The roots of the brachial plexus were exposed in the interscalene area through an incision along the sternomastoid muscle. The lesions of the C567 \pm C8 roots were confirmed. Availability of the ruptured roots was evaluated on the basis of their appearance at the intervertebral foramina, the response to stimulation of the branch to the serratus anterior and the appearance of the cross-section. In the beginning, the forearm nerve transfer was performed with a sterile tourniquet on the arm before the nerve transfers for elbow flexion was done. Later, the sequence was reversed so that the forearm procedure was done last with infiltration of saline-adrenaline $(1: 500,000)$ along the incision.

The radial nerve was exposed in the proximal forearm by tracing the superficial branch to its origin. The branch to the ECRB and the distal continuation as the posterior interosseous nerve were examined. The deficit in wrist dorsiflexion could be confirmed by the lack of response on stimulation of the ECRB branch. This branch was divided at its origin and turned distally ( - Video 1 ).

\section{Video 1}

Recipient technique. Online content including video sequences viewable at: https://www.thieme-connect. com/products/ejournals/html/10.1055/s-0040-1708114.

The incision was, then, extended distally. In the initial cases, one long curved incision was made with the distal portion along the volar ulnar aspect. The plane between the superficialis and profundus tendons was developed from the ulnar side and the anterior interosseous nerve was accessed between the flexor digitorum profundus (index finger slip) and the flexor pollicis longus. It was traced into the pronator quadratus muscle and divided as distally as possible. The nerve was traced proximally; care was taken to protect the branches to the flexor pollicis longus and to the flexor digitorum profundus. The origin of these branches served as the pivot point ( - Video 2 ).

\section{Video 2}

Donor technique. Online content including video sequences viewable at: https://www.thieme-connect.com/products/ejournals/ html/10.1055/s-0040-1708114. 
Access to the superficial plane was achieved by creating a tunnel through the flexor digitorum sublimus muscle taking care to avoid injury to the median nerve. The distal end of the AIN was, then, delivered proximally along this passage. In the more recent patients, the anterior interosseous nerve was reached via an incision along the anterolateral aspect of the forearm from the elbow to the wrist. The plane between the brachioradialis and the radial vessels was developed and the flexor pollicis longus was retracted to expose the AIN at its entry to the $P Q$. The proximal dissection of the nerve was facilitated by progressive division of the insertion of the flexor digitorum superficialis (FDS) muscle at the radius. This was continued till the pronator teres insertion. The nerve was divided distally and turned proximally; care was taken to protect the branches to the flexor pollicis longus and the flexor digitorum profundus (index finger slip). The FDS was reattached after repair of the AIN to the ECRB branch of the radial nerve. In this way, a tunnel through the muscle is not necessary and the innervation of the FDS is preserved.

Direct approximation of the two nerve ends was easily achieved in each case and the repair was performed using $9 / 0$ nylon sutures under the operating microscope. The arm was supported in an elbow pouch sling for four weeks after the operation. There was no tension on the repair site and a separate support for the wrist was not necessary as the repair site was not affected by wrist motion.

\section{Postoperative Assessment}

Each patient was examined every 3 months by the senior author with mean follow-up of 21 months (range 11.5-45 months). During pronation against resistance we observed for contractions of the ECRB, and when ECRB contractions become distinguishable patients were instructed that pronation produced wrist extension and they should practice motion at home without special physiotherapy training. During active wrist extension and finger flexion, wrist extension strength was assessed manually using the British Medical Research Council strength scale. ${ }^{6}$ Also, pronation, fingers flexion, and fingers extension power grading were documented.

\section{Statistical Analysis}

Data were verified, coded by the researcher, and analyzed using IBM-SPSS 21.0 (IBM-SPSS Inc., Chicago, Illinois, United States). Descriptive statistics-means, standard deviations, medians, ranges, and percentages-were calculated. Test of significances-chi-square test analysis-was performed to compare the proportions of the qualitative data of the MRC strength scale categories. A significant $p$-value was considered when it is equal or less than 0.05 .

\section{Ethical Considerations}

Approval for this study was obtained from the ethics committee of Faculty of Medicine, Aswan University, prior to study execution. In addition, all participants received a written consent form. The informed consent was clear and indicated the purpose of the study and their freedom to participate or withdraw at any time without any obligation. Furthermore, participants' confidentiality and anonymity were ensured by assigning each participant with a code number for the purpose of analysis only. The study was not based on any incentives or rewards for the participants.

\section{Results (-Video 3)}

\section{Video 3}

Results example. Online content including video sequences viewable at: https://www.thieme-connect.com/products/ejournals/ html/10.1055/s-0040-1708114.

- Table 1 and -Fig. 1 showed the baseline characteristics of the study cohort. The age of the studied cases ranged between 21 and 49 years, with a mean of 30.2 years. Moreover, the average preoperative delay was 4.7 months with a median of 4.5 (1.5-9 months). Also, the median follow-up time was 19 months (11.5-45 months), with a mean of 21 months.

In 17 of the 20 patients (85\%), wrist extension scored M4 while in the other three cases (15\%) it scored M3, with full range of wrist extension achieved in all patients. Interestingly, in those three cases with M3 wrist extension, the motor power of the thumb and index DIP flexion preoperatively was recorded only as M3, which may indicate weakness of the donor nerve (-Table 2 ).

Pronation power grading for 14 (70\%) patients was M4, the same as preoperative scoring, but in four cases the pronation downgraded from M4 to M3, in one case the pronation was lost to become $\mathrm{M} 0$, and in one case the forearm was stiff in mid-prone before and after surgery.

Regarding the thumb and index DIP flexion power scale, no cases have been downgraded due to the use of continuation of the AIN; meanwhile, power grading in $12(60 \%)$ of the 20 cases have been upgraded as a result of spontaneous recovery.

Also, at the last follow-up, the strength of finger extension improved (which could be explained by improvement of function in the incompletely injured C8 root territory) in $14(70 \%)$ patients and scored the same as the level before

Table 1 Baseline characteristics of the studied sample

\begin{tabular}{|l|l|l|}
\hline Variable & Category & $\boldsymbol{n}=\mathbf{2 0}$ \\
\hline Age in years & $\begin{array}{l}\text { Mean } \pm \text { standard } \\
\text { deviation }\end{array}$ & $30.15 \pm 9.2$ \\
\cline { 2 - 3 } & Median (range) & $25.5(21-49)$ \\
\hline $\begin{array}{l}\text { Preoperative Delay/ } \\
\text { months }\end{array}$ & $\begin{array}{l}\text { Mean } \pm \text { standard } \\
\text { deviation }\end{array}$ & $4.73 \pm 2.4$ \\
\cline { 2 - 3 } Follow-up/months & $\begin{array}{l}\text { Median (range) } \\
\text { Mean } \pm \text { standard }\end{array}$ & 21.5 (1.5-9) \\
\cline { 2 - 3 } & Meviation & $19(11.5-45)$ \\
\hline
\end{tabular}




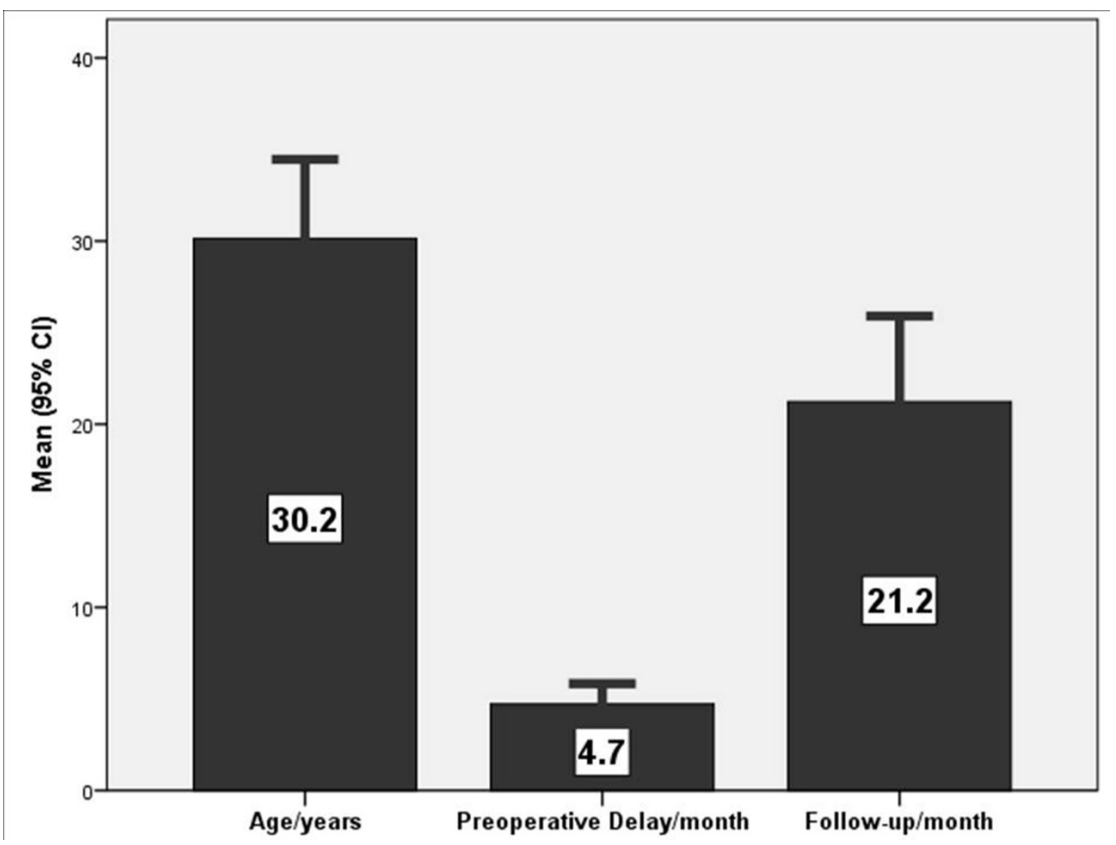

Fig. 1 Baseline characteristics of the studied sample.

Table 2 MRC strength scale preoperative versus postoperative comparisons $(n=20)$

\begin{tabular}{|c|c|c|c|}
\hline & Preoperative (M0-5) & Postoperative (M0-5) & $P$-value ${ }^{a}$ \\
\hline \multirow[t]{6}{*}{ Pronation } & $0(0 \%)$ & $1(5 \%)$ & \multirow[t]{6}{*}{$=0.047$} \\
\hline & $0(0 \%)$ & $0(0 \%)$ & \\
\hline & $0(0 \%)$ & $0(0 \%)$ & \\
\hline & $0(0 \%)$ & $4(20 \%)$ & \\
\hline & $19(95 \%)$ & $14(70 \%)$ & \\
\hline & $1(5 \%)$ & $1(5 \%)$ & \\
\hline \multirow[t]{5}{*}{ Wrist extension } & $20(100 \%)$ & $0(0 \%)$ & \multirow[t]{5}{*}{$=0.044$} \\
\hline & $0(0 \%)$ & $0(0 \%)$ & \\
\hline & $0(0 \%)$ & $0(0 \%)$ & \\
\hline & $0(0 \%)$ & $3(15 \%)$ & \\
\hline & $0(0 \%)$ & 17 (85\%) & \\
\hline \multirow[t]{6}{*}{ Thumb, index, DIP flexion } & $0(0 \%)$ & $0(0 \%)$ & \multirow[t]{6}{*}{$=0.032$} \\
\hline & $0(0 \%)$ & $0(0 \%)$ & \\
\hline & $0(0 \%)$ & $0(0 \%)$ & \\
\hline & $6(30 \%)$ & $0(0 \%)$ & \\
\hline & $10(50 \%)$ & $5(25 \%)$ & \\
\hline & $4(20 \%)$ & 15 (75\%) & \\
\hline \multirow[t]{6}{*}{ Finger extension } & $1(5 \%)$ & $1(5 \%)$ & \multirow[t]{6}{*}{$=0.039$} \\
\hline & $0(0 \%)$ & $0(0 \%)$ & \\
\hline & $1(5 \%)$ & $0(0 \%)$ & \\
\hline & $6(30 \%)$ & $0(0 \%)$ & \\
\hline & $10(50 \%)$ & $5(25 \%)$ & \\
\hline & $2(10 \%)$ & $14(70 \%)$ & \\
\hline
\end{tabular}

Abbreviations: MRC, British Medical Research Council strength scale DIP, distal interphalangeal joint. ${ }^{a}$ Chi-square test was used to compare the proportion difference between groups.

surgery in the other 6 (30\%) cases. In one patient, there was no change in the complete deficit in extrinsic extension noted before surgery.
The differences in the postoperative improvement proportion were demonstrated in - Table 2 . The proportion of improvement after surgery was statistically 
Table 3 Relationship between preoperative pronation, thumb, index, DIP flexion and postoperative wrist extension $(n=20)$

\begin{tabular}{|c|c|c|c|}
\hline & \multicolumn{2}{|c|}{ PO Wrist Extension } & \multirow[t]{2}{*}{$p$-Value } \\
\hline & 3 & 4 & \\
\hline \multirow[t]{2}{*}{ Preoperative Pronation } & $3(15 \%)$ & $16(80 \%)$ & \multirow[t]{2}{*}{$=0.850^{*}$} \\
\hline & $0(5 \%)$ & $1(5 \%)$ & \\
\hline \multirow[t]{3}{*}{ Preoperative Thumb, Index, DIP Flexion } & $3(15 \%)$ & $3(15 \%)$ & \multirow[t]{3}{*}{$=0.032^{* *}$} \\
\hline & $0(0 \%)$ & $10(50 \%)$ & \\
\hline & $0(0 \%)$ & $4(20 \%)$ & \\
\hline
\end{tabular}

*Fisher's exact test was used to compare the proportion difference between groups.

${ }^{* *}$ Chi-square test was used to compare the proportion difference between groups.

significant regarding the pronation; wrist extension; thumb, index, and DIP flexion; and finger extension $(p<0.05)$.

- Table 3 showed the relationship between preoperative pronation; thumb, index, and DIP flexion; and postoperative wrist extension. The strength of pronation noted before surgery did not help to predict successful restoration of wrist extension as this association was statistically not significant $(p<0.05)$, while the associations between the preoperative thumb and index DIP flexion and the post-operative wrist extension were statistically significant $(p<0.05)$.

\section{Discussion}

Paralysis pattern in brachial plexus injuries regarding the injured roots, while widely known and classified, ${ }^{7}$ is still a matter of debate with an estimated incidence of $21 \%$ for the upper middle traumatic brachial plexus injuries. ${ }^{8}$ Bertelli et al revisited this classification in their studies and tried to change the concept that C5-C7 pattern has loss of extension in elbow, wrist, and thumb. They assumed that most of the cases which are usually diagnosed as a $\mathrm{C} 5-\mathrm{C} 7$ are actually C5-C8 lesions and they called this pattern as "T1 hand."

In this $\mathrm{C} 5-\mathrm{C} 8$ pattern they observed that wrist extensors are always paralyzed, but wrist extension is preserved in half of the patients by the finger extensors action. ${ }^{5}$ However, this compensation for wrist extension by finger extensors is not functional as with finger flexion in the grasp action ( - Video 4 ) the wrist drops because there is no active wrist extensor to maintain the wrist in extension. And it is well known that when the wrist is flexed, grasp power is significantly weakened. ${ }^{9}$ Also, half of the C5-C8 cases in their study have retained triceps function, but in only half of them are M3 or better strength preserved. ${ }^{5}$

The senior author has observed some spontaneous return of the pectoralis major and triceps over a year. Hence, we do not address those deficits in the primary nerve operation

\section{Video 4}

Finger extension compensation. Online content including video sequences viewable at: https://www.thieme-connect. com/products/ejournals/html/10.1055/s-0040-1708114. while wrist extension always requires attention. Although the $\mathrm{C} 5-\mathrm{C} 6$ root lesions are clearly defined regarding the neurological deficits and have a well-known algorithm of distal nerve transfers with predictable good results, the $\mathrm{C} 5-\mathrm{C} 7$ or $\mathrm{C} 5-\mathrm{C} 8$ root injuries have poorer results of shoulder and elbow distal nerve transfers with unclear algorithm to deal with the wrist extension deficit.

So, the usual reconstruction protocol for $\mathrm{C} 5-\mathrm{C} 7$ or $\mathrm{C} 5-\mathrm{C} 8$ root injuries gives the priority to the shoulder and elbow primary nerve reconstruction, leaving the wrist extension deficit for a tendon transfer reconstruction in a second stage procedure. The available donor options for tendon transfer or nerve transfer in those cases is limited in comparison to the usual wrist and fingers extension reconstruction of the radial nerve palsy, ${ }^{5}$ as the pronator teres is paralyzed and the flexor carpi ulnaris is not intact in all cases. So, the alternative is to reconstruct the wrist extension by flexor digitorum superficialis tendon transfer or nerve transfer. ${ }^{4}$

However, the strength of finger flexion is already weaker in such patients (grasp strength $34 \%$ and pinch $40 \%$ of the opposite normal hand according to Bertelli. ${ }^{5}$ Hence, harvesting a slip of the flexor digitorum superficialis carries the risk of weakening the grip strength further. ${ }^{5}$ We prefer to address the deficits at the shoulder, elbow and wrist in the primary operation at an early stage.

The timing of the primary operation is still a point of contention. Some surgeons prefer to observe for improvement in the function of the finger flexors and intrinsics with a view to utilize nerve transfers from the median and/or ulnar nerves to restore elbow flexion. One can be guided by the evidence of root avulsions that can be provided by the high quality of imaging currently available.

Before 2013 the senior author's policy for reconstructing wrist extension deficit in $\mathrm{C} 5-\mathrm{C} 7$ or $\mathrm{C} 5-\mathrm{C} 8$ root injuries was to do secondary tendon transfer of the flexor digitorum superficialis of the middle finger to the ECRB. This procedure was usually performed after restoration of strong elbow flexion in a second stage. Then after 2013, he had shifted to systematic use of the neurotization of the ECRB with the terminal portion of the anterior interosseous nerve in the same surgical setting of shoulder and elbow nerve reconstruction. The integrity of the donor nerve was checked in our series preoperatively by the thumb and index DIP flexion and the pronation power. 
It is worthy to note that all our cases reported as $\geq \mathrm{M} 4$ in pronation, and in 14 cases thumb and index DIP flexion reported as $\geq \mathrm{M} 4$ and in the other 6 cases as M3. This may lead us to consider M4 power of the thumb and index DIP flexion as a minimum prerequisite to do AIN to ECRB nerve transfer to ensure good-quality donor nerve and hence good wrist extension results as the forearm pronation power may be deceiving due to the pronation effect of the flexor mass of the forearm which may mask the weak and poorly innervated pronator quadratus. Also, the pronation effect of the flexor mass of the forearm gave us the confidence not to reinnervate the pronator quadratus muscle after taking its nerve as a donor in our series (unlike Bertlli's study in which he used a distal motor branch of the FDS to the index finger to neurotize the distal branch of the AIN in 19 cases of his 28 cases series).

This distal motor branch of the FDS to the index finger to the distal branch of the AIN was described by Tung and Mackinnon. ${ }^{13}$ Another compensation for pronation loss is through shoulder internal rotation by the action of pectoralis major and latissmus dorsi. In such patients with extensive affection of pectoralis major and latissmus dorsi without recovery, perhaps it would be prudent to add a nerve transfer for the pectoralis major. That involves use of an intercostal nerve for one of the pectoral branches and does not interfere with spontaneous recovery in the future. Another possibility that deserves trial is reinnervation of the serratus anterior and pectoralis major (by intercostal nerve transfers) and later shoulder fusion (when elbow flexion and wrist dorsiflexion have been restored). In this series, the pronation in $21 \%$ of the cases has been downgraded ( 4 cases, 3 of them downgraded from M4 to M3 and only 1 case downgraded from M4 to M0) after surgery, which matches Bertelli's results ( 6 out of 28 cases [21.4\%]).

In spite of not doing reinnervation of the pronator quadratus in our study, the postoperative pronation results were comparable to Bertelli's results, which may make this reinnervation procedure questionable. But we think that regaining the wrist extension is more prioritized than the pronation. Overall results of wrist extension recovery in our series were 85\% M4 and 15\% M3 which matches with Bertelli's results (90\% M4). Comparing PQ to ECRB nerve transfer results with the flexor digitorum superficialis nerve transfer to ECRB, only three cases were reported by Bertelli to have poor results, with two cases that scored M3 and 1 case M3 with no independent wrist extension or full range of extension in any case. ${ }^{14}$

Another two cases of flexor digitorum superficialis nerve transfer to ECRB were reported by Ukrit et al, with M4 wrist extension but both of their cases were C5-C6-C7 injuries with intact or recovering $\mathrm{C} 8$ root. ${ }^{15}$

In comparison with secondary reconstruction with tendon transfer the advantages of PQ to ECRB nerve transfer are, first, to avoid a second stage surgery and hence decrease rehabilitation time and, second, save the available tendons (FCU or FDS) for another recipient like finger extension, if needed.

\section{Conclusion}

The PQ to ECRB nerve transfer in C5-C7 or C5-C8 brachial plexus injuries for independent wrist extension reconstruction gives consistently good results with minimal donor morbidity. The function appears simultaneously with the elbow flexion and the period of rehabilitation gets shortened. The option of a secondary tendon transfer is not affected, and the available donor tendons can be reserved for fingers extension augmentation if needed.

\section{Conflict of Interest}

None.

\section{Acknowledgments}

\section{References}

1 Abid A. Brachial plexus birth palsy: management during the first year of life. Orthop Traumatol Surg Res 2016;102(1(Suppl): S125-S132

2 Li G, Shao L. An innovative robotic training system imitating the cervical spine behaviors during rotation-traction manipulation. Robot Auton Syst 2018;107(1):116-128

3 Ramachandran S, Midha R. Recent advances in nerve repair. Neurol India 2019;67(7, Supplement) :S106-S114

4 Bincaz LE, Cherifi H, Alnot JY. Palliative tendon transfer for reanimation of the wrist and finger extension lag. Report of 14 transfers for radial nerve palsies and ten transfers for brachial plexus lesions [in French]. Chir Main 2002;21(1):13-22

5 Bertelli JA, Tacca CP, Winkelmann Duarte EC, Ghizoni MF, Duarte $\mathrm{H}$. Transfer of the pronator quadratus motor branch for wrist extension reconstruction in brachial plexus palsy. Plast Reconstr Surg 2012;130(6):1269-1278

6 Vanhoutte EK, Faber CG, van Nes SI, et al; PeriNomS Study Group. Modifying the Medical Research Council grading system through Rasch analyses. Brain 2012;135(Pt 5) :1639-1649

$7 \mathrm{Kim}$ DH, Murovic JA, Tiel RL, Kline DG. Mechanisms of injury in operative brachial plexus lesions. Neurosurg Focus 2004;16(5):E2

8 Faglioni W Jr, Siqueira MG, Martins RS, Heise CO, Foroni L. The epidemiology of adult traumatic brachial plexus lesions in a large metropolis. Acta Neurochir (Wien) 2014;156(5):1025-1028

9 LiZM. The influence of wrist position on individual finger forces during forceful grip. J Hand Surg Am 2002;27(5):886-896

10 Birch R. Timing of surgical reconstruction for closed traumatic injury to the supraclavicular brachial plexus. J Hand Surg Eur Vol 2015;40(6):562-567

11 Kato N, Htut M, Taggart M, Carlstedt T, Birch R. The effects of operative delay on the relief of neuropathic pain after injury to the brachial plexus: a review of 148 cases. J Bone Joint Surg Br 2006;88(6):756-759

12 Doi K, Otsuka K, Okamoto Y, Fujii H, Hattori Y, Baliarsing AS. Cervical nerve root avulsion in brachial plexus injuries: magnetic resonance imaging classification and comparison with myelography and computerized tomography myelography. J Neurosurg 2002;96(3, Suppl) :277-284

13 Tung TH, Mackinnon SE. Flexor digitorum superficialis nerve transfer to restore pronation: two case reports and anatomic study. J Hand Surg Am 2001;26(6):1065-1072

14 Bertelli JA, Ghizoni MF. Transfer of a flexor digitorum superficialis motor branch for wrist extension reconstruction in C5-C8 root injuries of the brachial plexus: a case series. Microsurgery 2013;33(1):39-42

15 Ukrit A, Leechavengvongs S, Malungpaishrope K, Uerpairojkit C, Chongthammakun S, Witoonchart K. Nerve transfer for wrist extension using nerve to flexor digitorum superficialis in cervical 5, 6, and 7 root avulsions: anatomic study and report of two cases. J Hand Surg Am 2009;34(9):1659-1666 